Sains Malaysiana 49(1)(2020): 121-128

http://dx.doi.org/10.17576/jsm-2020-4901-14

\title{
Stress and Food Intake among University Students - Is There a Relationship?
}

(Stres dan Pengambilan Makanan dalam Kalangan Pelajar Universiti - Apakah Perkaitannya?)

\author{
Shi-Hui Cheng* \& Muhammad Khair Mohd KamiL
}

\section{ABSTRACT}

There is a growing interest in the effects of stress on food consumption. Studies suggest that stress could either increases or decreases food consumption among university students. As the prevalence of stress is evident among university students in various countries, this study aims to investigate the relationship of stress on dietary consumption among undergraduate students of a private university. A cross-sectional study was carried out and a total of 100 participants aged between 18-25 years and non-smokers were recruited. Anthropometric data was measured and participants were asked to answer a questionnaire which accessed their sociodemographic background, stress level, frequency of food intake and 3-day dietary intake. The data collected were then analysed using SPSS software. This study found that males consumed cooked vegetables less frequently than females (male $=2.38 \pm 1.48$; female $=3.68 \pm 1.31, p<0.001$ ) while consumed soft drinks more frequently than females (male $=1.90 \pm 1.31$; female $=1.28 \pm 1.24, p<0.05$ ). In addition, stressed group consume significantly lower energy, fat (including saturated fat, monounsaturated fatty acids and polyunsaturated fatty acids) and calcium than non-stressed group $(p<0.05)$. In conclusion, the prevalence of stress among university students in this study was $40 \%$. Students eat lesser when they are in stress and this could negatively impact their health. Therefore, stress management interventions need to be implemented to help the university students to manage their stress and this could help to promote healthy eating behaviour among university students.

Keywords: Dietary intake; eating behavior; food consumption; stress; university students

\section{ABSTRAK}

Pada masa kini, minat untuk mendalami kesan tekanan ke atas pemakanan seseorang individu semakin meluas. Beberapa kajian mendapati bahawa tekanan akan meningkatkan atau mengurangkan pola pemakanan seseorang. Oleh kerana prevalens tekanan semakin meningkat dalam kalangan pelajar universiti di pelbagai negara, maka kajian ini bertujuan untuk mengkaji hubungan tekanan terhadap pola pemakanan dalam kalangan pelajar di sebuah universiti swasta. Kajian keratan lintang telah dijalankan dan seramai 100 peserta yang berumur antara 18-25 tahun dan bukan perokok menyertai kajian ini. Data antropometri diukur dan para peserta diminta menjawab soal selidik yang mengumpulkan data mengenai latar belakang mereka, kadar tekanan yang dialami, kekerapan pengambilan makanan dan rekod pengambilan makanan selama 3 hari. Data yang dikumpul kemudian dianalisis manggunakan perisian SPSS. Kajian ini mendapati bahawa wanita lebih kerap memakan sayur-sayuran berbanding dengan lelaki (lelaki=2.38 1.48 ; wanita $=3.68 \pm 1.31, p<0.001)$ dan lelaki lebih kerap meminum minuman ringan berbanding dengan wanita (lelaki= $1.90 \pm 1.31$; wanita $=1.28 \pm 1.24, p<0.05$ ). Di samping itu, pengambilan makanan daripada segi tenaga, lemak dan kalsium bagi kumpulan yang tertekan lebih rendah berbanding kumpulan yang tidak tertekan (p<0.05). Secara kesimpulannya, prevalens tekanan dalam kalangan pelajar universiti dalam kajian ini adalah 40\%. Pengambilan makanan dalam kalangan pelajar yang tertekan berkurangan dan ini memberi kesan buruk kepada kesihatan mereka. Oleh itu, intervensi pengurusan tekanan perlu dilaksanakan untuk membantu pelajar universiti menguruskan tekanan mereka dan ini dapat membantu meningkatan pola pemakanan yang sihat dalam kalangan pelajar universiti.

Kata kunci: Corak pemakanan; pelajar universiti; pengambilan makanan; pola pemakanan; tekanan

\section{INTRODUCTION}

Stress is defined as an organism's physiological response to counter threatening or demanding circumstances that trigger multiple defense mechanisms. The body responds to stress in two ways; through the autonomic nervous system which triggers the fight or flight response which is immediate and through the activation of HypothalamicPituitary-Adrenal (HPA) axis that leads to a cascade of events producing glucocorticoid (Pariante et al. 2008). In acute effects of stress on appetite, corticotrophin-releasing hormone (CRH) inhibits neuropeptide Y (NPY)/ agoutirelated peptide (AGRP) neurons in the arcuate nucleus of the hypothalamus. Hence, this reaction causes appetite suppression after an acute stress period (Currie 2003). On the other hand, prolonged stress causes the body to use a lot of energy to cope with it. Hence, glucocorticoid serum 
level is elevated to allow energy replacement causing multiple metabolic reactions which includes one that stimulates appetite (Sominsky 2014).

Prevalence of stress among university students should be given extra attention as it was reported that in Malaysia, the prevalence of stress was $36 \%$ (Gan et al. 2011) while in Turkey, Hong Kong and Australia was 27\%, 43\% and 52.9\%, respectively (Bayram et al. 2008; Papier et al. 2015; Wong et al. 2006). It has also been linked with obesity and academic performance (Sominsky et al. 2014). University students are susceptible to changes in eating behaviour due to several factors including stress. Young adults were reported to have poor dietary practices as they leave home for college. This allows them to have autonomy in making food choices which are also influenced by the availability of food and socioeconomic status (Gan et al. 2011). Hence, skipping breakfast and opting for fast foods are common among college students. The situation is made worse as they experience academic stresses from projects, competitiveness and academic overload. Other factors like body dissatisfaction, low self-esteem (Thomas et al. 2010), weight teasing and pressure to be thin (Benas et al. 2010) also play a role in college students risks of stress.

In studies that consider the apparent effects of stress on eating practices, they found that psychological distress contributes to changes in eating behaviour (Gan et al. 2011). It either increases or decreases food consumption as a whole or increases consumption of certain types of food and at the same time decreases consumption of the other types. Zellner et al. (2006) reported that participants had a higher intake of high-fat and high-calorie snack foods during stressful periods to make them feel better. Oliver et al. (1999) found that the quantity of unhealthy foods consumed increases while the quantity of healthy foods consumed decreases under stress conditions. Consumption of processed foods, meat alternatives and alcohol increased among stressed groups whereas intake of vegetables and fruits tend to decrease (Papier et al. 2015). Meanwhile, other study concluded food consumption to deplete under stress conditions (Cartwright et al. 2003).

Differences in response to stress may be influenced by an individual's dieting status. Restrained eating refers to the voluntary control to restrict food intake for the weight loss purposes. Restrained eaters tend to consume more food while non-restrained eaters decreasing the food intake under stress condition (Wardle et al. 2000). Restrained eaters were also found to report hyperphagic response during stress more commonly as compared to nonrestrained eaters (Wallis \& Hetherington 2004). Another factor such as gender has also been found to have effects on food choices under stress conditions. Hallam et al. (2016) reported women are more likely to increase consumption of chocolate and sweets and reduce consumption of fish and meat and fruit and vegetables compared to men. Stressed women tend to consume unhealthier food compared to non-stressed women while stressed men tend to consume lower amounts of unhealthy food than non-stressed men (Zellner et al. 2006). Variability in results could be due to multiple factors including differences in an individual's response to stress with some being hyperphagic while others being hypophagia (Yau \& Potenza 2013). Difficulty in obtaining precise food intake also contributes to the outcome variability (Oliver et al. 1999).

While studies in areas concerning stress and its influences on food consumption has gained attention in various countries like United Kingdom, Australia, United States, Canada and Germany (Emond et al. 2016; Oliver et al. 1999; Papier et al. 2015; Sharma et al. 2010; Zellner et al. 2007), little has been done in Malaysia to investigate these variables among university students. Previous studies in Malaysia focus on the eating disorder behavior and the level of stress among university students (Gan et al. 2011; Ganasegeran et al. 2012; Saat et al. 2014), however, the relationship between stress and food intake among university students remains unclear. Thus, this study aims to investigate the relationship between stress and food intake among undergraduate students of a private university in Malaysia.

\section{Materials AND Methods}

\section{PARTICIPANTS AND SAMPLING}

This was a cross-sectional study conducted among private university students. By using $\mathrm{G}^{*}$ power software for sample size calculation, parameters of 0.5 effect size, $5 \%$ margin of error, power $0.8,100$ respondents were required. A total of 100 university students from a private university were enrolled using simple random sampling. Inclusion criteria were undergraduate students aged between 18-25 years and non-smokers. This study was approved by the University Science and Engineering Research Ethics Committee (MK-20170429).

\section{ANTHROPOMETRIC MEASUREMENT}

Height of the participants was measured using a SECA 217 stadiometer to the nearest $0.1 \mathrm{~cm}$ while their weight and Body Mass Index (BMI) were measured using Bioelectrical Impedance Analysis (Tanita DC-430 MA) with shoes removed. The World Health Organization (WHO) BMI cutoff points were used in this research.

\section{QUESTIONNAIRE AND DATA COLLECTION}

The questionnaire was divided into four sections; sociodemographic background, perceived stress scale, food frequency questionnaire and three-day dietary record.

\section{SOCIODEMOGRAPHIC BACKGROUND}

Among the data collected were age, gender, ethnicity, year of study and school/faculty. To differentiate between restrained and non-restrained eaters, the question 'Are you 
trying to lose weight at present' was included. Physical activity levels were also accessed.

\section{PERCEIVED STRESS SCALE}

Cohen's Perceived Stress Scale (Cohen et al. 1983) was used to measure individuals stress perception. Ten items pertaining their feelings and thoughts in the past month were listed with answer options of 0 (Never), 1 (Almost never), 2 (sometimes), 3 (fairly often) and 4 (very often). Participants with a total score of $\leq 20$ were grouped as 'non-stressed' and those who scored $\geq 21$ were grouped as 'stressed'.

\section{FOOD FREQUENCY QUESTIONNAIRE}

The food frequency questionnaire (FFQ) contains a list of 12 different food groups used to access the frequency of consumption of the different type of foods by individuals. Each food group rated on a 6-point Likert scale ranging from 'never (0)', 'once a month (1)', 'once a week (2)', '2-4 times a week (3)', '5-6 times a week (4)', to 'daily (5)'.

\section{THREE-DAY DIETARY RECORD}

A three-day dietary record was attached. Participants were asked to fill up their food intake on a weekend and two weekdays to represent their normal diet. Types of food eaten and their quantity consumed from breakfast until supper was recorded.

\section{Data ANALYsis}

Recorded food and nutrient intake were first analyzed using Nutritics nutrition analysis software version 5.0. Statistical analysis was carried out using Statistical Package for the Social Sciences (SPSS) version 24.0. Independent samples t-test were used to compare between numerical variables and the genders. To compare between two categorical variables, Chi-square test was conducted. A p-value of $<0.05$ is considered significant.

\section{RESULTS}

\section{SOCIODEMOGRAPHIC CHARACTERISTICS}

Sociodemographic characteristics of the participants are presented in Table 1. A total of 100 university students took part in this study with a mean age of $21.27 \pm 1.50$ years. The three major races in Malaysia (Malay, Indian and Chinese) made up $40 \%, 23 \%$ and $9 \%$ of the total participants, respectively, while the remaining $28 \%$ were made up of students from different ethnic backgrounds such as Pakistani, Punjabi, Kazakh, Arab, African and British. Majority of the participants were third-year undergraduates
(37\%). Most male students were from Arts (32\%) and Engineering Faculty (32\%) while females were mostly from the Faculty of Science (70\%). Slightly more females were reported to be on diet (46\%) as compared to males (36\%). Males were generally more active than females with $82 \%$ reported to be moderate or highly active compared to their counterparts $(68 \%)$. Males were also generally heavier than females, having a mean of $70.88 \pm 12.55 \mathrm{~kg}$ while females $53.8 \pm 9.84 \mathrm{~kg}(\mathrm{p}<0.001)$. The trend is similar for heights with females having shorter mean height $1.57 \pm 0.05 \mathrm{~m}$ while males $1.72 \pm 0.05 \mathrm{~m}$ $(p<0.001)$. Majority of the participants were within the normal BMI range. Out of all the participants, $40 \%$ of the students were identified as stressed while $60 \%$ were nonstressed. However, there was no significant difference in perceived stress between genders.

\section{INTAKE FREQUENCY OF DIFFERENT FOOD GROUPS}

Differences in the intake frequency of cooked vegetables and soft drinks were found (Table 2). Male consumed cooked vegetables less frequently $(2.38 \pm 1.48)$ in the past one month as compared to females $(3.68 \pm 1.31)$. In the soft drinks category, males were found to consume more frequently as compared to females $(p<0.05)$ with the mean frequency of $1.90 \pm 1.31$ and $1.28 \pm 1.24$ among respective genders. Contrary to expectations, there was no significant difference for all food categories between the non-stressed and stressed groups.

\section{NUTRIENT INTAKE BETWEEN MALES AND FEMALES}

Table 3 shows the comparison of average nutrient intake between males and females. Male participants were found to consume higher energy in a day $(1815.91 \mathrm{kcal} /$ day $)$ than female participants $(1510.03 \mathrm{kcal} / \mathrm{day})(\mathrm{p}<0.01)$. Carbohydrate intake was also found to differ between the genders with males consuming more carbohydrate (220.92 $\mathrm{g}$ /day) than females $(176.86 \mathrm{~g} /$ day $)(\mathrm{p}<0.01)$. Significant difference of protein intake was also reported between genders. Mean protein intake among males (90.68 g/day) was higher than mean protein intake among females (69.51 $\mathrm{g} /$ day) $(\mathrm{p}<0.001)$. For micronutrients, significant differences were found in daily intakes of potassium and vitamin D $(p<0.01$ and $p<0.05$, respectively). No significant difference was found in the intake of other nutrients.

\section{NUTRIENT INTAKE BETWEEN STRESSED AND NON-STRESSED GROUP}

Table 4 shows the comparison of nutrient intake between the stressed and non-stressed groups. Stressed group was found to consume lower energy intake (1579 kcal/day) as compared to non-stressed group (1718 kcal/day). Fat intake is higher in the non-stressed group (62.09 g/day) as compared to the stressed group (56.44 g/day). Saturated fat and mono-unsaturated fatty acid (MUFA) intakes showed similar trends $(\mathrm{p}<0.01)$. Poly-unsaturated fatty acid (PUFA) 
TABLE 1. Sociodemographic characteristics of respondents by genderv

\begin{tabular}{|c|c|c|c|c|c|c|c|}
\hline \multirow[t]{2}{*}{ Characteristics } & \multicolumn{2}{|c|}{$\begin{array}{c}\text { Total } \\
(n=100)\end{array}$} & \multicolumn{2}{|c|}{$\begin{array}{c}\text { Male } \\
(\mathrm{n}=50)\end{array}$} & \multicolumn{2}{|c|}{$\begin{array}{l}\text { Female } \\
(\mathrm{n}=50)\end{array}$} & \multirow[t]{2}{*}{ p-value } \\
\hline & $\mathrm{n}$ & $\%$ & $\mathrm{n}$ & $\%$ & $\mathrm{n}$ & $\%$ & \\
\hline Age (y)(mean/SD) & \multicolumn{2}{|c|}{$21.27 \pm 1.50$} & \multicolumn{2}{|c|}{$21.38 \pm 1.65$} & \multicolumn{2}{|c|}{$21.16 \pm 1.33$} & 0.465 \\
\hline $\begin{array}{l}\text { Ethnicity } \\
\text { Malay } \\
\text { Indian } \\
\text { Chinese } \\
\text { Others }\end{array}$ & $\begin{array}{c}40 \\
9 \\
23 \\
28\end{array}$ & $\begin{array}{c}40 \\
9 \\
23 \\
28\end{array}$ & $\begin{array}{c}15 \\
5 \\
6 \\
24\end{array}$ & $\begin{array}{l}30 \\
10 \\
12 \\
48\end{array}$ & $\begin{array}{c}25 \\
4 \\
17 \\
4\end{array}$ & $\begin{array}{c}50 \\
8 \\
34 \\
8\end{array}$ & $0.001 * * *$ \\
\hline $\begin{array}{l}\text { Year of Study } \\
\text { Foundation } \\
1^{\text {st }} \text { year } \\
2^{\text {nd }} \text { year } \\
3^{\text {rd }} \text { year } \\
4^{\text {th }} \text { year }\end{array}$ & $\begin{array}{c}9 \\
30 \\
22 \\
37 \\
2\end{array}$ & $\begin{array}{c}9 \\
30 \\
22 \\
37 \\
2\end{array}$ & $\begin{array}{c}7 \\
15 \\
11 \\
15 \\
2\end{array}$ & $\begin{array}{c}14 \\
30 \\
22 \\
30 \\
2\end{array}$ & $\begin{array}{c}2 \\
15 \\
11 \\
22 \\
0\end{array}$ & $\begin{array}{c}4 \\
30 \\
22 \\
44 \\
0\end{array}$ & 0.192 \\
\hline $\begin{array}{l}\text { Faculty } \\
\text { Foundation } \\
\text { Arts } \\
\text { Engineering } \\
\text { Science }\end{array}$ & $\begin{array}{l}10 \\
26 \\
19 \\
45\end{array}$ & $\begin{array}{l}10 \\
26 \\
19 \\
45\end{array}$ & $\begin{array}{c}8 \\
16 \\
16 \\
10\end{array}$ & $\begin{array}{l}16 \\
32 \\
32 \\
20\end{array}$ & $\begin{array}{c}2 \\
10 \\
3 \\
35\end{array}$ & $\begin{array}{c}4 \\
20 \\
6 \\
70\end{array}$ & $0.001 * * *$ \\
\hline $\begin{array}{l}\text { Trying to lose weight } \\
\text { Yes } \\
\text { No }\end{array}$ & $\begin{array}{l}41 \\
59\end{array}$ & $\begin{array}{l}41 \\
59\end{array}$ & $\begin{array}{l}18 \\
32\end{array}$ & $\begin{array}{l}36 \\
64\end{array}$ & $\begin{array}{l}23 \\
27\end{array}$ & $\begin{array}{l}46 \\
54\end{array}$ & 0.309 \\
\hline $\begin{array}{l}\text { Physical Activity } \\
\text { Low } \\
\text { Moderate } \\
\text { High }\end{array}$ & $\begin{array}{l}25 \\
65 \\
10\end{array}$ & $\begin{array}{l}25 \\
65 \\
10\end{array}$ & $\begin{array}{c}9 \\
33 \\
8\end{array}$ & $\begin{array}{l}18 \\
66 \\
16\end{array}$ & $\begin{array}{c}16 \\
32 \\
2\end{array}$ & $\begin{array}{c}32 \\
64 \\
4\end{array}$ & 0.062 \\
\hline $\begin{array}{l}\text { Height (m) } \\
(\text { mean/SD) }\end{array}$ & & & & & & & $0.001 * * *$ \\
\hline $\begin{array}{l}\text { Weight (kg) } \\
(\text { mean/SD) }\end{array}$ & & & & & & & $0.001 * * *$ \\
\hline $\begin{array}{l}\text { BMI } \\
(\text { mean/SD) }\end{array}$ & & & & & & & $0.001 * * *$ \\
\hline $\begin{array}{l}\text { BMI category } \\
\text { Underweight }(<18.5) \\
\text { Normal }(18.5-24.9) \\
\text { Overweight }(25-29.9) \\
\text { Obese }(>29.9)\end{array}$ & $\begin{array}{c}11 \\
65 \\
18 \\
6\end{array}$ & $\begin{array}{c}11 \\
65 \\
18 \\
6\end{array}$ & $\begin{array}{c}1 \\
33 \\
12 \\
4\end{array}$ & $\begin{array}{c}2 \\
66 \\
24 \\
8\end{array}$ & $\begin{array}{c}10 \\
32 \\
6 \\
2\end{array}$ & $\begin{array}{c}20 \\
64 \\
12 \\
4\end{array}$ & 0.286 \\
\hline $\begin{array}{l}\text { Stress condition } \\
\text { Stress } \\
\text { Non-stress }\end{array}$ & $\begin{array}{l}40 \\
60\end{array}$ & $\begin{array}{l}40 \\
60\end{array}$ & $\begin{array}{l}18 \\
32\end{array}$ & $\begin{array}{l}36 \\
64\end{array}$ & $\begin{array}{l}22 \\
28\end{array}$ & $\begin{array}{l}44 \\
56\end{array}$ & 0.414 \\
\hline
\end{tabular}

\section{DiscusSION}

intake among non-stressed participants was also higher than stressed participants $(p<0.05)$. Calcium was also consumed more by non-stressed group as compared to stressed group $(\mathrm{p}<0.01)$. These differences suggest that food consumption of stressed group is significantly lower than non-stressed group. However, nutrient intake among restrained and non-restrained eaters under stressed conditions has found no statistical significance (Table 5). This shows that diet status of individual does not affect their food intake when in stress.
Our study showed that male participants consumed higher calorie, protein and carbohydrates as compared to female participants. As commonly known, men eat relatively more than women as they have higher daily requirements of energy intake and also in proportion of their heavier body weight (Oliver et al. 2000). Women, in particular, are more health-conscious and are more likely to avoid certain types of food for weight loss and health reasons (Wardle et al. 2004). Our study found that males tend to consume cooked vegetables less frequently as compared to females 
TABLE 2. Frequency of food intake in a month among students by gender and stress condition

\begin{tabular}{|c|c|c|c|c|c|c|}
\hline & $\begin{array}{c}\text { Male }(n=50) \\
\text { Mean } \pm \text { SD }\end{array}$ & $\begin{array}{c}\text { Female }(\mathrm{n}=50) \\
\text { Mean } \pm \mathrm{SD}\end{array}$ & p-value & $\begin{array}{c}\text { Non-Stress }(n=60) \\
\text { Mean } \pm \text { SD }\end{array}$ & $\begin{array}{c}\text { Stress }(n=40) \\
\text { Mean } \pm \text { SD }\end{array}$ & P-value \\
\hline Sweets & $2.28 \pm 1.55$ & $2.20 \pm 1.34$ & 0.783 & $2.11 \pm 1.41$ & $2.42 \pm 1.48$ & 0.298 \\
\hline Cakes/Cookies & $2.00 \pm 1.17$ & $1.92 \pm 0.92$ & 0.706 & $2.13 \pm 0.98$ & $1.70 \pm 1.11$ & 0.430 \\
\hline Snacks & $2.40 \pm 1.45$ & $2.64 \pm 1.20$ & 0.372 & $2.46 \pm 1.37$ & $2.60 \pm 1.29$ & 0.628 \\
\hline Fast foods & $2.10 \pm 1.26$ & $1.80 \pm 0.96$ & 0.186 & $2.08 \pm 1.07$ & $1.75 \pm 1.19$ & 0.150 \\
\hline Fresh fruits & $2.38 \pm 1.24$ & $2.86 \pm 1.19$ & 0.052 & $2.55 \pm 1.32$ & $2.72 \pm 1.10$ & 0.491 \\
\hline Salad & $1.70 \pm 1.32$ & $1.84 \pm 1.51$ & 0.625 & $1.70 \pm 1.31$ & $1.87 \pm 1.57$ & 0.549 \\
\hline Cooked Vegetables & $2.38 \pm 1.48$ & $3.68 \pm 1.31$ & $0.001 * * *$ & $3.05 \pm 1.58$ & $3.00 \pm 1.48$ & 0.875 \\
\hline Soft Drinks & $1.90 \pm 1.31$ & $1.28 \pm 1.24$ & $0.017^{*}$ & $1.63 \pm 1.26$ & $1.52 \pm 1.39$ & 0.688 \\
\hline Meat & $4.14 \pm 1.22$ & $3.80 \pm 1.19$ & 0.164 & $4.01 \pm 1.25$ & $3.90 \pm 1.17$ & 0.641 \\
\hline Fish & $2.00 \pm 1.30$ & $2.04 \pm 1.26$ & 0.877 & $1.91 \pm 1.38$ & $2.17 \pm 1.10$ & 0.325 \\
\hline Milk Products & $3.16 \pm 1.44$ & $3.38 \pm 1.42$ & 0.446 & $3.31 \pm 1.46$ & $3.20 \pm 1.39$ & 0.692 \\
\hline Cereal/Cereal products & $2.06 \pm 1.55$ & $2.26 \pm 1.58$ & 0.526 & $2.26 \pm 1.53$ & $2.00 \pm 1.61$ & 0.408 \\
\hline
\end{tabular}

$* \mathrm{p}<0.05, * * * \mathrm{p}<0.001$

TABLE 3. Comparison of average 3 days nutrient intake between males and females

\begin{tabular}{|c|c|c|c|c|}
\hline Macronutrients & $\begin{array}{c}\text { Overall } n=100 \\
\text { mean } \pm \mathrm{SD}\end{array}$ & $\begin{array}{l}\text { Male } \mathrm{n}=50 \\
\text { mean } \pm \mathrm{SD}\end{array}$ & $\begin{array}{c}\text { Female } \mathrm{n}=50 \\
\text { mean } \pm \mathrm{SD}\end{array}$ & p-value \\
\hline Energy (kcal/d) & $1662.97 \pm 518.23$ & $1815.91 \pm 576.96$ & $1510.03 \pm 402.46$ & $0.003 * *$ \\
\hline Carbohydrate (g/d) & $198.89 \pm 75.24$ & $220.93 \pm 85.39$ & $176.87 \pm 56.18$ & $0.003 * *$ \\
\hline Protein (g/d) & $80.09 \pm 31.98$ & $90.68 \pm 36.25$ & $69.51 \pm 22.88$ & $0.001 * * *$ \\
\hline Fat $(g / d)$ & $59.83 \pm 21.56$ & $62.69 \pm 23.07$ & $56.98 \pm 19.76$ & 0.187 \\
\hline Fibre (g/d) & $10.36 \pm 4.27$ & $10.26 \pm 3.77$ & $10.47 \pm 4.75$ & 0.808 \\
\hline $\operatorname{Sugar}(g / d)$ & $45.50 \pm 29.02$ & $47.60 \pm 30.75$ & $43.40 \pm 27.34$ & 0.472 \\
\hline Saturated Fat (g/d) & $15.51 \pm 7.66$ & $16.68 \pm 7.46$ & $14.33 \pm 7.74$ & 0.126 \\
\hline MUFA (g/d) & $11.59 \pm 6.30$ & $12.00 \pm 7.25$ & $11.18 \pm 5.25$ & 0.651 \\
\hline $\operatorname{PUFA}(\mathrm{g} / \mathrm{d})$ & $6.23 \pm 3.98$ & $5.98 \pm 3.84$ & $6.47 \pm 4.13$ & 0.447 \\
\hline Cholesterol (mg/d) & $240.31 \pm 156.30$ & $249.76 \pm 168.14$ & $230.84 \pm 144.59$ & 0.548 \\
\hline \multicolumn{5}{|l|}{ Micronutrients } \\
\hline Sodium (mg/d) & $769.99 \pm 542.53$ & $791.16 \pm 521.11$ & $748.81 \pm 567.63$ & 0.698 \\
\hline Potassium (mg/d) & $1586.01 \pm 674.33$ & $1785.81 \pm 750.06$ & $1386.20 \pm 524.10$ & $0.003 * *$ \\
\hline Calcium (mg/d) & $432.87 \pm 379.01$ & $451.36 \pm 439.19$ & $414.37 \pm 310.87$ & 0.628 \\
\hline Phosphorus (mg/d) & $730.47 \pm 429.00$ & $755.68 \pm 476.08$ & $705.25 \pm 379.33$ & 0.559 \\
\hline Magnesium (mg/d) & $111.36 \pm 67.65$ & $111.17 \pm 77.22$ & $111.55 \pm 57.55$ & 0.850 \\
\hline Iron (mg/d) & $9.12 \pm 6.97$ & $9.38 \pm 8.51$ & $8.86 \pm 5.06$ & 0.705 \\
\hline Vitamin D $(\mu \mathrm{g} / \mathrm{d})$ & $1.91 \pm 1.71$ & $1.56 \pm 1.32$ & $2.22 \pm 1.96$ & $0.021 *$ \\
\hline
\end{tabular}

MUFA $=$ monounsaturated fatty acid, PUFA $=$ polyunsaturated fatty acid

$*$ p-value $<0.05, * *$ p-value $<0.01, * * *$ p-value $<0.001$

$(\mathrm{p}<0.001)$ and consume soft drinks more frequently than females $(p<0.05)$. This is in line with the outcome of several other studies. Rehm et al. (2008) reported higher consumption of soft drinks among adult men in New York City as compared to females. A study conducted in Germany also found women to be more health-conscious that they choose healthier food types than men (Sharma et al. 2010).
Ganasegeran et al. (2012) also reported that majority of the medical students in Malaysia consumed fruits less than three times per week and half of them had fried food at least twice per week.

Interestingly, when comparing for differences in the amount of nutrients consumed, this study showed that non-stressed students consume more foods which are high 
TABLE 4. Comparison of average 3-days nutrient intake among stressed and non-stressed students

\begin{tabular}{lcccc}
\hline Macronutrients & Overall & Non-Stress & Stress & p-value \\
& $\mathrm{n}=100$ & $\mathrm{n}=60$ & $\mathrm{n}=40$ & mean $\pm \mathrm{SD}$ \\
\hline Energy $(\mathrm{kcal} / \mathrm{d})$ & $1662.97 \pm 518.23$ & $1718.89 \pm 578.61$ & $1579.11 \pm 404.02$ & $0.042^{*}$ \\
Carbohydrate $(\mathrm{g} / \mathrm{d})$ & $198.89 \pm 75.24$ & $208.16 \pm 82.50$ & $184.99 \pm 61.18$ & 0.215 \\
Protein $(\mathrm{g} / \mathrm{d})$ & $80.09 \pm 31.98$ & $80.52 \pm 32.91$ & $79.46 \pm 30.93$ & 0.939 \\
Fat $(\mathrm{g} / \mathrm{d})$ & $59.83 \pm 21.56$ & $62.09 \pm 24.27$ & $56.44 \pm 16.41$ & $0.022^{*}$ \\
Fibre $(\mathrm{g} / \mathrm{d})$ & $10.36 \pm 4.27$ & $10.34 \pm 4.32$ & $10.40 \pm 4.24$ & 0.334 \\
Sugar $(\mathrm{g} / \mathrm{d})$ & $45.50 \pm 29.02$ & $46.26 \pm 30.74$ & $44.35 \pm 26.59$ & 0.813 \\
Saturated Fat $(\mathrm{g} / \mathrm{d})$ & $15.51 \pm 7.66$ & $16.83 \pm 8.65$ & $13.53 \pm 5.38$ & $0.005^{* *}$ \\
MUFA $(\mathrm{g} / \mathrm{d})$ & $11.59 \pm 6.30$ & $12.16 \pm 7.06$ & $10.76 \pm 4.93$ & $0.009 * *$ \\
PUFA $(\mathrm{g} / \mathrm{d})$ & $6.23 \pm 3.98$ & $6.51 \pm 4.51$ & $5.81 \pm 3.05$ & $0.031^{*}$ \\
Cholesterol $(\mathrm{mg} / \mathrm{d})$ & $240.31 \pm 156.30$ & $251.73 \pm 166.78$ & $223.16 \pm 139.41$ & 0.191 \\
Micronutrients & & & & 0.132 \\
Sodium $(\mathrm{mg} / \mathrm{d})$ & $769.99 \pm 542.53$ & $779.35 \pm 564.38$ & $755.93 \pm 514.73$ & 0.414 \\
Potassium $(\mathrm{mg} / \mathrm{d})$ & $1586.01 \pm 674.33$ & $1602.76 \pm 720.85$ & $1560.88 \pm 605.91$ & $0.002^{* *}$ \\
Calcium $(\mathrm{mg} / \mathrm{d})$ & $432.87 \pm 379.01$ & $494.80 \pm 455.80$ & $339.98 \pm 188.71$ & 0.485 \\
Phosphorus $(\mathrm{mg} / \mathrm{d})$ & $730.47 \pm 429.00$ & $737.70 \pm 469.16$ & $719.63 \pm 366.07$ & 0.113 \\
Magnesium $(\mathrm{mg} / \mathrm{d})$ & $111.36 \pm 67.65$ & $113.50 \pm 76.97$ & $108.22 \pm 51.68$ & 0.121 \\
Iron $(\mathrm{mg} / \mathrm{d})$ & $9.12 \pm 6.97$ & $9.99 \pm 8.45$ & $7.81 \pm 3.50$ & 0.838 \\
Vitamin D $(\mu \mathrm{g} / \mathrm{d})$ & $1.91 \pm 1.71$ & $1.91 \pm 1.70$ & $1.91 \pm 1.74$ & \\
\hline
\end{tabular}

MUFA $=$ monounsaturated fatty acid, PUFA $=$ polyunsaturated fatty acid $*$ p-value $<0.05, * *$ p-value $<0.01$

TABLE 5. Comparison of average 3-days nutrient intake among stressed restrained eaters and non-restrained eaters

\begin{tabular}{lcccc}
\hline Macronutrients & Overall & Restrained & Non-restrained & p-value \\
& $\mathrm{n}=40$ & $\mathrm{n}=21$ & $\mathrm{n}=19$ & mean $\pm \mathrm{SD}$ \\
\hline Energy $(\mathrm{kcal} / \mathrm{d})$ & $1579.11 \pm 404.02$ & $1575.24 \pm 402.01$ & $1583.37 \pm 417.19$ & 0.956 \\
Carbohydrate $(\mathrm{g} / \mathrm{d})$ & $184.99 \pm 61.18$ & $180.01 \pm 62.14$ & $190.50 \pm 61.29$ & 0.747 \\
Protein $(\mathrm{g} / \mathrm{d})$ & $79.46 \pm 30.93$ & $78.26 \pm 28.67$ & $80.79 \pm 34.00$ & 0.307 \\
Fat $(\mathrm{g} / \mathrm{d})$ & $56.44 \pm 16.41$ & $59.85 \pm 14.47$ & $52.68 \pm 17.95$ & 0.504 \\
Fibre $(\mathrm{g} / \mathrm{d})$ & $10.40 \pm 4.24$ & $11.41 \pm 3.93$ & $9.28 \pm 4.37$ & 0.942 \\
Sugar $(\mathrm{g} / \mathrm{d})$ & $44.35 \pm 26.59$ & $43.74 \pm 25.77$ & $45.03 \pm 28.15$ & 0.870 \\
Saturated Fat $(\mathrm{g} / \mathrm{d})$ & $13.53 \pm 5.38$ & $13.91 \pm 5.41$ & $13.11 \pm 5.45$ & 0.700 \\
MUFA $(\mathrm{g} / \mathrm{d})$ & $10.76 \pm 4.93$ & $11.45 \pm 5.02$ & $10.00 \pm 4.83$ & 0.841 \\
PUFA $(\mathrm{g} / \mathrm{d})$ & $5.81 \pm 3.05$ & $6.44 \pm 2.85$ & $5.12 \pm 3.19$ & 0.603 \\
Cholesterol $(\mathrm{mg} / \mathrm{d})$ & $223.16 \pm 139.41$ & $227.51 \pm 128.69$ & $218.36 \pm 153.82$ & 0.528 \\
Micronutrients & & & & 0.643 \\
Sodium $(\mathrm{mg} / \mathrm{d})$ & $755.93 \pm 514.73$ & $830.93 \pm 598.07$ & $673.04 \pm 403.49$ & 0.946 \\
Potassium $(\mathrm{mg} / \mathrm{d})$ & $1560.88 \pm 605.91$ & $1658.45 \pm 596.25$ & $1453.03 \pm 614.02$ & 0.548 \\
Calcium $(\mathrm{mg} / \mathrm{d})$ & $339.98 \pm 188.71$ & $336.60 \pm 157.19$ & $343.71 \pm 222.87$ & 0.051 \\
Phosphorus $(\mathrm{mg} / \mathrm{d})$ & $719.63 \pm 366.07$ & $653.63 \pm 268.36$ & $792.57 \pm 446.79$ & 0.374 \\
Magnesium $(\mathrm{mg} / \mathrm{d})$ & $108.22 \pm 51.68$ & $108.51 \pm 51.30$ & $107.90 \pm 53.50$ & 0.359 \\
Iron $(\mathrm{mg} / \mathrm{d})$ & $7.81 \pm 3.50$ & $7.28 \pm 2.97$ & $8.39 \pm 3.99$ & 0.476 \\
Vitamin D $(\mu \mathrm{g} / \mathrm{d})$ & $1.91 \pm 1.74$ & $2.01 \pm 2.03$ & $1.79 \pm 1.40$ & \\
\hline
\end{tabular}

MUFA $=$ monounsaturated fatty acid, PUFA= polyunsaturated fatty acid 
in energy and fat (based on a higher fat, saturated fat, MUFA and PUFA intake) as compared to stressed students. Although previously mentioned studies reported the opposite (stressed people tends to choose fatty foods more than non-stressed people), this result is supported by findings reported by Zellner et al. (2007) where nonstressed group were found to eat more of unhealthier food than the stressed group. Stone et al. (1994) also found 72\% of adults were hypophagic when in stress as compared to $28 \%$ of those who were hyperphagic. A research piloted by Jeong et al. (2013) also found that stress reduces food intake that it contributes to weight loss. They reported that during a period of stress, hypothalamic genes expression which contributes to weight control is affected. At the same time, expression of ghrelin and pro-opiomelanocortin is altered and so do corticosterone and leptin levels which gradually increases and decreases, respectively. Besides that, this trend could also be influenced by the habit of skipping meals among stressed individuals with females normally reporting a higher prevalence of skipping breakfast (Cartwright et al. 2003). Oliver et al. (1999) added decrease in fish and meat consumption among stressed people. Reduced food intake among stress participants could possibly lead to reduced intake of energy, fat, saturated fat, MUFA, PUFA and calcium as portrayed in this study.

Comparison between genders showed a significant difference in frequency of food consumption between males and females while there is no significant difference between them under a stressed condition. The possible explanation could be deduced from differences in food intake between genders under stress condition. Men and women respond to stress in different ways. When compared at gender level, stressed females tend to eat more than their counterpart. This was proven through various studies which look into gender differences when stress. Oliver et al. (1999), reported that women were more hyperphagic than men under stress and there was an increase of food consumption in stressed women and a significant decrease of food consumption in stressed men. Zellner et al. (2006) reported different eating patterns between genders where $46 \%$ of women reported overeating than $17 \%$ men and more men (54\%) reported undereating than women (37\%). Oliver et al. (2000) also mentioned that meals consumed by emotional eaters whom mostly are women are highly energy-dense as compared to those consumed by low emotional eaters (mainly men). Also, the tendency of women to control their high-fat food intake when not in stress allows them to increase their food intake when in stress as they lose control of themselves during this period (Zellner et al. 2006). The bidirectional response towards stress perhaps answers the insignificant differences in frequency of food consumption between genders under a stressed condition.

The habit of avoiding unhealthy foods among individuals under normal circumstances causes them to have a high tendency of disinhibition during the stress period. Several studies concluded that restrained eaters/ dieters are hyperphagic in response to stress while nonrestrained eaters/non-dieters are either hypophagic or maintain normal intake under stress (Wallis \& Hetherington 2004; Yau \& Potenza 2013). However, current study found no differences in food consumption between restrained eaters and non-restrained eaters. Despite the contrary findings against aforementioned studies and counter to initial expectations, similar findings were reported by Oliver et al. (2000). They found no significant effects of diet status on overall food intake (neither in weight nor energy content) and even when food intake was analysed in the form of energy percentage from carbohydrate, protein, and fat. Barker et al. (2015) also found no relationship between dietary restraint and response to food intake due to examination stress. Perhaps assessment of diet status through the question 'Are you trying to lose weight at present?' only caters to the dieting status and did not take into account the susceptibility to dietary disinhibition. Also, a psychometric measure could have been used to measure restrained eating to better compare results with laboratory studies (Oliver et al. 1999). Yau and Potenza (2013) also reported that in addition to diet status, type of stress also influences an individual's eating behaviour. As such, non-restrained eaters tend to eat less while restrained eaters do not significantly eat more in response to physical stress. On the contrary, emotional threat causes restrained eaters to increase food intake but does not necessarily cause non-restrained eaters to suppress their food intake (Wallis \& Hetherington 2004). Unfortunately, types of stressors were not determined in this study and this may have contributed to the result generated among stressed participants.

Having mentioned all the findings, this study also has its limitations. It is worth to acknowledge the difficulty faced when collecting reliable and valid food intake information in this study as it is solely dependent on the participant's memory and honesty to report their dietary intake. Hence, the accuracy of food intake in terms of the amount and the types of food consumed is compromised. Besides that, the causal effects of stress on eating practices were difficult to be determined in this study due to its nature as a cross-sectional study. In addition, this study also did not take into account other possible factors that may affect stress-induced eating behaviour such as availability of food, peer pressure, knowledge and socioeconomic status of participants.

\section{CONCLUSION}

The prevalence of stress among university students in this study was $40 \%$, thus shows that students are prone to stress. The key finding in this study was that food consumption among students was indeed altered under stressed conditions with stressed students eating lesser than nonstressed students. Stressed group consume significantly lower energy, fat (including saturated fat, MUFA, PUFA) and calcium than non-stressed group. This should not be 
taken lightly as nutrient deficiency, in the long run, will impose negative health impacts on students and their performance. Future studies should explore other possible contributors between stress and food intake. Differences in response towards stress between genders could also be detrimental to better understand stress impacts on individuals. Finally, stress management interventions should be implemented to reduce stress and further promote good eating practices among the university students.

\section{REFERENCES}

Barker, M.E., Blain, R.J. \& Russell, J.M. 2015. The influence of academic examinations on energy and nutrient intake in male university students. Nutrition Journal 14: 98.

Bayram, N. \& Bilgel, N. 2008. The prevalence and sociodemographic correlations of depression, anxiety and stress among a group of university students. Social Psychiatry and Psychiatric Epidemiology 43: 667-672.

Benas, J.S., Uhrlass, D.J. \& Gibb, B.E. 2010. Body dissatisfaction and weight-related teasing: A model of cognitive vulnerability to depression among women. Journal of Behavior Therapy and Experimental Psychiatry 41: 352-356

Cartwright, M., Wardle, J., Streggles, N., Simon, A.E., Croker, H. \& Jarvis, M.J. 2003. Stress and dietary practices in adolescents. Health Psychology 22: 362.

Cohen, S., Kamarck, T. \& Mermelstein, R. 1983. A global measure of perceived stress. Journal of Health and Social Behavior 24: 385-396.

Currie, P.J. 2003. Integration of hypothalamic feeding and metabolic signals: Focus on neuropeptide Y. Appetite 41: 335-337.

Emond, M., Ten Eycke, K., Kosmerly, S., Robinson, A.L., Stillar, A. \& Van Blyderveen, S. 2016. The effect of academic stress and attachment stress on stress-eaters and stress-undereaters. Appetite 100: 210-215.

Gan, W.Y., Mohd Nasir, M.T., Zalilah, M.S. \& Hazizi, A.S. 2011. Direct and indirect effects of sociocultural influences on disordered eating among Malaysian male and female university students. A mediation analysis of psychological distress. Appetite 56: 778-783.

Ganasegeran, K., Al-Dubail, S., Qureshi, A.M., Al-Abed, A.A., Rizal, A.M. \& Aljunid, S.M. 2012. Social and psychological factors affecting eating habits among university students in a Malaysian medical school: A crosssectional study. Nutrition Journal 11: 48.

Hallam, J., Boswell, R.G., DeVito, E.E. \& Kober, H. 2016. Gender-related differences in food craving and obesity. The Yale Journal of Biology and Medicine 89(2): 161-173.

Jeong, J.Y., Lee, D.H. \& Kang, S.S. 2013. Effects of chronic restraint stress on body weight, food intake, and hypothalamic gene expressions in mice. Endocrinology and Metabolism 28: 288-296.

Oliver, G. \& Wardle, J. 1999. Perceived effects of stress on food choice. Physiology and Behavior 66: 511-515.

Oliver, G., Wardle, J. \& Gibson, E.L. 2000. Stress and food choice: A laboratory study. Psychosomatic Medicine 62: 853-865.

Papier, K., Ahmed, F., Lee, P. \& Wiseman, J. 2015. Stress and dietary behaviour among first-year university students in Australia: Sex differences. Nutrition 31: 324-330.
Pariante, C.M. \& Lightman, S.L. 2008. The HPA axis in major depression: Classical theories and new developments. Trends in Neurosciences 31: 464-468.

Rehm, C.D., Matte, T.D., Van Wye, G., Young, C. \& Frieden, T.R. 2008. Demographic and behavioral factors associated with daily sugar-sweetened soda consumption in New York City adults. Journal of Urban Health 85: 375-385.

Saat, N.Z.M., Chew, C.L., Divya, V., Intan Hafizah, I., Lee, P.L., Mohd Ramadan, A.H., Ooi, H.Y., Seah, J.L. \& Yap, W.J. 2014. Relationship of stress on the eating behaviors of science undergraduates in Kuala Lumpur. Research Journal of Applied Sciences, Engineering and Technology 7(8): 1668-1676.

Sharma, B., Harker, M., Harker, D. \& Reinhard, K. 2010. Youth transition to university in Germany and Australia: An empirical investigation of healthy eating behaviour. Journal of Youth Studies 13: 353-367.

Sominsky, L. \& Spencer, S.J. 2014. Eating behavior and stress: A pathway to obesity. Frontiers in Psychology 5: 434.

Stone, A.A. \& Brownell, K. 1994. The stress-eating paradox: Multiple daily measurements in adult males and females. Psychology and Health 9: 425-436.

Thomas, J., Khan, S. \& Abdulrahman, A.A. 2010. Eating attitudes and body image concerns among female university students in the United Arab Emirates. Appetite 54: 595-598.

Wallis, D.J. \& Hetherington, M.M. 2004. Stress and eating: The effects of ego-threat and cognitive demand on food intake in restrained and emotional eaters. Appetite 43(1): $39-46$.

Wardle, J., Steptoe, A., Oliver, G. \& Lipsey, Z. 2000. Stress, dietary restraint and food intake. Journal of Psychosomatic Research 48(2): 195-202.

Wardle, J., Haase, A.M., Steptoe, A., Nillapun, M., Jonwutiewes, K. \& Bellisle, F. 2004. Gender differences in food choice: The contribution of health beliefs and dieting. Annals of Behavioral Medicine 27: 107-116.

Wong, J.G., Cheung, E., Chan, K.C. \& Tang, S.W. 2006. Webbased survey of depression, anxiety and stress in firsttertiary tertiary education students in Hong Kong. The Australian and New Zealand Journal of Psychiatry 40(9): 777-782.

Yau, Y.H. \& Potenza, M.N. 2013. Stress and eating behaviors. Minerva Endocrinologica 38(3): 255-267.

Zellner, D.A., Loaiza, S., Gonzalez, Z., Pita, J., Morales, J., Pecora, D. \& Wolf, A. 2006. Food selection changes under stress. Physiology and Behavior 87: 789-793.

Zellner, D.A., Saito, S. \& Gonzalez, J. 2007. The effect of stress on men's food selection. Appetite 49: 696-699.

School of Biosciences

University of Nottingham Malaysia

Jalan Broga

43500 Semenyih, Selangor Darul Ehsan

Malaysia

*Corresponding author; email: shihui.cheng@nottingham.edu. my

Received: 20 September 2019

Accepted: 11 October 2019 